\title{
THE SOCIAL INDIVIDUAL.
}

BY PROFESSOR A. T. ORMOND.

Princetun University

The consciousness of the present is distinctively social and tends to the merging of the individual more or less completely in the social organism. This tendency itself is no doubt onesided, and there are dangers with which it is threatened. It arose, however, as a healthy reaction against the separative and antisocial tendencies of the thought of the eighteenth century which had embodied themselves in extreme forms and in which the divine right of the isolated individual had become a central dogma. Modern psychology was born on the rising tide of this movement, and has not as yet quite lost the impress of its parentage. Until a comparatively recent date the struggling science might very appropriately and not unjustly have been styled the psychology of the solitary.

Now apart from external motives there may be found in consciousness itself a degree of justification for this separative tendency. If we look into the workings of our own consciousness through the spectacles of the most advanced psychology we will find that it is possible for us to distinguish what we may call our isolated, individual self, from a larger self that includes our social relationships. Thus from the former point of view I conceive myself simply as a solitary ego or a me standing over against the other me of my family and social environment, and, in a sense, as having interests that are distinct from theirs, while from the point of view of the social relationships this dualism seems to be transcended, and the me becomes husband and father in relation to the family, and citizen in relation to the community and nation. It was the solitary ego rather than the me of the social relationships that became the subject of the old psychology, and the new psychology has arisen as the result of an 
effort to cure this one-sidedness and to restore the broader social self to its rights. The project of the new psychology thus contemplates the reform of the psychological doctrine of the self as a basis for an ultimate reform of the doctrine of social relations.

How then, we may ask, is this new concept of the self as a socius to be achieved? In the first place, it is clear that it will be necessary to deny the validity of the older point of view in which the self stood apart from its social relationships and viewed these as external to its own interests and self-activities. On this point the old psychology can derive little support from the ordinary consciousness of the plain man to which its appeal was so commonly made; for the plain man's self is one that includes all his possessions, so that even an insult to his dog is taken as an indignity to himself. The real self is the concrete self of the social relationships; the self that can say, " nothing that touches any of my possessions can be indifferent to me." It is the business of the new psychology to show the validity of this by exhibiting either analytically, that the stripping off of the social relations leaves a mutilated ego, and when carried far enough nothing that is definable; or, genetically, that it is one and the same consciousness and life history in which are developed the representation of the individual self and that of the social other, and that the distinction between these two representations is intra rather than ultra to the real self. What then do we mean by the self as a socizes, and how is the concept of the socizs to be scientifically grounded? The answer will involve a reflection in two parts, the first dealing analytically, the second genetically with the problem. In the first place, then, we may ask for an analytic answer to the question what the notion of the self as a socius is. Let us take as our point of departure the consciousness of an adult, say that of an intelligent man who is at the same time innocent of psychology and not much given to selfrefiection. Take, for example, the ordinary man of business and society whose life is absorbed in outer activities, and let our analysis proceed from the standpoint of his own conscious relation to his activities rather than from the aloofness of a mere spectator. His world must be represented, in fact, as one in which 
his own aggressive and organizing agency stands central and to which every part of it will be related. Let such a man begin to inspect his own conscious processes; or, what would be still better, let some one who is trained in this species of analysis enter into his point of view as far as may be possible and perform the work of analysis in his behalf. If the man be primarily a man of business and only in a secondary sense a votary of society, it will be found that the standpoint from which he is most accustomed to consider himself and the issues of his life is that of his business relations, and that proceeding out from these he derelops a conscious representation of himself as so bound up with a community of other selves of the same type, and whose point of view is identical with his own, as to give rise to a whole system of responses in the form of demands and obligations. This system of responses will constitute what is most real in his life, and were he to attempt to form any construct of himself as he would be apart from these vital relations of the business world, he would either find the enterprise impossible, or the self he would achieve would be hypothetical rather than real. The real self is the self of vital interests, and apart from this there can be no real self. The only resource open to the man in question, if he be not satisfied with his business self, is to transfer his vital interests to some other world. Let this be the world of society. Here it will be found that the same drama repeats itself; his vital responses take on the society order, and when he attempts to dissociate himself from his societ $y$ relations the self that remains is mutilated and to a great degree divested of reality. This analysis may be carried through the whole sphere of his social relationships so as to include the domestic, civic and religious, and the same conclusions will be found to hold true. The self-consciousness of the family man is that of the individual clothed with a specification, so that the real self is now father, husband or son, and this specification thus modifies and determines the basis of all his conscious responses and consequently the whole sphere of his conscious responsibilities, privileges and enjoyments. Again the civic consciousness by virtue of which he becomes a citizen, a patriot and a member of a political party, is the bearer of a still further 
specification of the central self. The citizen-consciousness is that of the conscious self specified and defined in the direction of the civic interests and relationships, and thus becoming the bearer of a larger complex of duties, privileges, responsibilities, rights and enjoyments. Lastly his religious consciousness by virtue of which he becomes a worshipper of God is a still further specification in view of his sense of unique relation to a being that transcends him. The result is a self defined and specified in this particular direction, and responding concretely to a system of motives that are distinctively religious; in short, a self that is not real apart from its religious relations.

We have only to follow out this analysis into every detail of life in order to reach the conviction that the self which is central in all these activities, and which we may, therefore, call the cardinal self, is not in any sense independent of its social relations, or in any sense complete without them. The social relations constitute in fact the modes by which the self passes from the stage of indeterminateness, where it only vaguely realizes itself, to that of more complete specification and definiteness, through which it becomes more completely self-realized. The socius is, therefore, the more fully defined and realized self. James, in his very suggestive chapter on The Consciousness of Self, in the second volume of his 'Psychology,' gives an exhibition of this analytic method ${ }^{1}$ and shows how the self achieves the various and successive stages of its definition in terms of the social medium. He represents these several stages as so many selves, and maintains that a man has a plurality of selves, each of which has its own characteristic ways of responding to its world. This may be accepted as a striking and, on the whole, appropriate way of stating the case, provided we do not go to the extreme which James avoids, and assert that these selves are not only distinguishable, but also separable. Our doctrine will lose coherence if we do not hold to it that it is the same cardinal self that is central and continuous in all this variation of form, and that the process as a whole is to be taken as the mode in which this cardinal self attains to definite and concrete self-consciousness.

1 Psychology, Vol. I., Chapter X. The Consciousness of Self. This chapter has marked an epoch in the recent psychology of Self.. 
Passing to the second method of dealing with the social aspect of the self, the genetic, we find important illustrations of it in the work that is being done in the field of genetic psychology. The general aim of genetic psychology is, of course, to discover and formulate the stages and conditions of the development of consciousness. But a special department of the science has arisen of late in response to a pressing demand for a more adequate treatment of the psychological aspects of the social consciousness. The result has been a group of works which have had for their aim the genetic study of the social individual or self. Taking the work of Baldwin as developed in his Social and Ethical Interpretations in Mental Development as representative of the common aim of these works, we may found on it the following representation. The problem of this branch of the genetic enterprise is to show how the social consciousness may be brought under the rubrics of psychological evolution so as to give a demonstration of the solidarity of the social with the consciousness of the individual self. And this aim is achieved by showing in detail how the self in coming to its own clear and definite self-apprehension is brought by the same process to a recognition of its social other. The investigations we have in mind posit, by implication at least, a germinal self or at least a consciousness of the self-type as the inner individual center of response, and the object is to exhibit the method and the environmental forces which lead this germinal self-consciousness through the progressive stages of a development in which the social becomes a corporate part of the very self. Now what is needed in order that this aim may be effected and the development be seen to be real is to determine first what is meant by social environment and heredity; (2) the characteristic form of reaction in this field, and (3) the kind of definition or specification that the self obtains as a result. In short, the categories of the evolution must be defined with reference to the kind of material in which they are supposed to work.

Now it is not difficult to determine the nature of the social environment. If we consider the self as a social unit in a system of interacting units, it will be clear that the environment is simply the social medium in which the organism exists and performs its 
functions, and that this medium not only includes the social individuals of the community, but also the social institutions and conventions of the community-life and conduct. Let us represent a child, for instance, as a floating center of adaptation in a medium that will embrace not only other social individuals and institutions, but will also hold in solution the whole current mass of conventions, convictions and tendencies that are characteristic of the time. This complex will represent the environment with which the child's consciousness will be in interactive relation. What, then, shall we designate as social heredity? It is possible, of course, that social modifications may be transmitted in a direct, organic way. But our opinion as to this would be largely determined by the theory of heredity which we regarded as nearest the truth. It is obvious that a Weissmanian could have little sympathy with the notion of the organic transmission of social effects. If, however, we recognize the superorganic character of the social, we will not be disposed to think it strange if we are asked to look in the super-organic field for the principle of the conservation of social effects. In truth, we have been asked, notably by Baldwin, to look into the heart of the social medium itself for this principle of conservation. When we consider this medium carefully we find that it not only contains a mass of what we may call social traditions in solution, but that there is a tendency in this medium for these traditions to embody themselves not only in institutions which perpetuate certain great ideas or trends of the mass, but also to give themselves an unorganized though well-defined form in what may be called the spirit, which the past has projected into the present. This spirit will manifest itself most broadly in civilizations, less broadly in national character, so far as it grows out of traditions. It will give itself more and more circumscribed but not less powerful embodiments in the traditional spirit of tribes, cliques, special institutions and families, the spirit of the family, for example, being one of the most potent educators of the child. The tendency of this conserving force is, therefore, toward the fixity of definite types in distinction from that of the environment, which is a medium in which everything tends to become fluent. Now it is to this conserving 
force, however it may express itself, whether in the perpetuity of institutions, the conservation of literature and art, or in the hereditary spirit of family, tribe and nation, that the name social heredity is to be applied, and it is evident that when we have overcome a little our biological prejudices against the superorganic in general we will be ready to admit that we have a force here which performs a real function of conservation and transmission. We shall take the liberty then to agree with those who have thus defined the principle of social heredity.

The second problem we have to determine is the form which the responsive, adaptive movement takes in this field. The psychologists to whom we have referred develop two lines of investigation which have a bearing on the question, the first of which has for its object the exhibition of the general method by which the subject-consciousness comes to a realization of itself and its world, while the second aims to determine the principle by means of which this result is achieved. Now in regard to the general method by which the subject-consciousness realizes its world, it has been carried almost to the point of demonstration, I think, that the movement is first objective. Consciousness goes out upon its objective world in some pulse of aggressive activity, and in this act is able in some way to penetrate and realize the object. This leads to a return reactive movement in which consciousness as the result of its penetration of its world, attains to a higher and better defined conception of itself. The general movement is thus circular and embraces objective and subjective stages. What then is the principle through which this movement realizes itself? Here again we come upon a super-organic phase of our problem. The principle or category which was first pointed out by Tarde, and developed by Baldwin, Royce and others, is that of imitation, a term that is somewhat difficult to define, but whose operation may be definitely conceived. Let us suppose that a boy of say six years, who is the son of a carpenter, after observing his father plane and fit together some flooring boards, procures a plane and some pieces of board and makes the effort to plane them and fit them together. ${ }^{1}$ The process is manifestly one of

1 This illustration is taken from an instance that actually happened a few days ago in connection with the buildiug of my own house. 
imitation, and the boy has the representation of his father's action as a copy which he is trying to reproduce. By a series of tentative movements let us suppose that the boy succeeds in a passable reproduction of the copy he has set before him. We have here not only a transaction, but an experience. The transaction is the imitative movement or series of movements by means of which the boy has reproduced a certain kind of effect in the objective world. The experience is the subjective reaction of this result, the modification or specification which the self has achieved when it has not only expressed the emotional exaltation which we call the feeling of success, but has also become defined by its knowledge of the feeling of a carpenter when he produces the original of the boy's copy. In other words, the boy has not only produced an effect in the objective world, but he has also defined a consciousness in himself analogrous to the consciousness which in his father accompanied the act of carpentry. And it is open to the analyst in this field to point out how this new consciousness becomes, by virtue of the fact that it takes the form of a defined idea, a motive impulse to further activities in the same line. We thus have exhibited the operation of a principle which tends to the repetition of activities on a progressively higher scale, and thus to the perfection of the adaptive result.

Let us now pass on to the third point, and consider the kind of modification or specification which the self receives as the result of this process. Referring once more to the case of the boy, it is clear that the knowledge of the way in which an objective act of skill is to be performed will not be the only respect in which his self-consciousness will become defined. More important than this in its psychological bearings will be the fact that through his activity the boy is able to enter into his father's consciousness and to realize, in fact, how a carpenter feels in connection with his work. In short, he has made an important step in the direction of mastering the carpenter's point of view from which he contemplates and reacts upon his world. We have, now, only to change the illustrations to forms that are more distinctively social, as, for example, the imitation by children of family, social or religious functions, in order to be able 
to see that this category of imitation stands as a definite mode, whether we regard it as the only mode or not, by and through which the growing consciousness not only makes progressive inroads into the objective, but also achieves a progressive definition and qualification of itself.

If now we take into account both lines of psychological investigation, we find that in both inquiries the social vindicates itself as an essential element in the defined consciousness of self. The analytic inquiry made this clear by showing that to strip off the social modification is also to take away the definitions of selfconsciousness, so that where the process has been completed there will remain nothing but the wholly undefined cardinal self which the whole investigation has presupposed. The various social selves are reducible, therefore, in the last analysis to phases of the one central self. The results of the genetic inquiry have been found to be on the whole confirmatory of the results of analysis. The problem here is one of history, and the aim is to show how the self develops its social character. The outcome of the investigation is, as we have seen, not only confirmatory of the result of analysis, but it teaches an impressive lesson in its own way. When we have followed the process by which the social elements gain entrance into the growing consciousness, and have seen that it is the very process also in which the self-consciousness becomes defined, our conviction becomes that of one who has been permitted to be present at a demonstration.

Admitting the truth of the doctrine as thus far developed it is still open to us to ask whether the boy's own subjective consciousness with which he accompanies the progressive stages of the objective activity is not his only immediate experience, and whether he does not learn how his father feels in a given situation, by traveling through that situation, and first learning how he himself feels?

This seems to be a more adequate view, and we are disposed to recant anything we have said to the contrary, and to put in its place the statement that the boy learns the true subjectivity of situations by traveling through them, and that being the model of his father traveling through the same situation in 
mind, the interpretation of the father's consciousness is the result of a largely spontaneous application of analogy. This will enable us to define the boy's relation to his model in a way that will save the initiative of his own consciousness, for if it turns out that there is only one way of getting at the inner consciousness of another, and that, by traveling through some objective movement in an imitative way which generates directly a modification in our consciousness which is referred to the consciousness of the other, through the model that connects it with the same kind of activity; if this proves to be true, then we are in possession of a datum that will be important when we come to determine how one conscious self may interact with another.

Analysis of the situation makes it evident that the above statement of the case is correct, and that while the boy seems to be reading his father's consciousness directly through his model, he is, in the first instance, determining his own consciousness by means of the imitative activity, and reaches the construct of his father's consciousness only by what we may venture to call an immediate analogical inference. If this be true, the question may arise as to the precise function which the model performs in the boy's development. The imitative function is clear enough, and there can be no question that what the boy has in the foreground of his consciousness is not simply a representation of a series of movements, but rather the representation of this series as connected with, and as being the movements of, a definite individual, his father. The whole model is, therefore, a representation of his father performing a series of movements and the boy's attempt to imitate the whole situation. It is clear then that the effort to imitate is in reality an effort on the part of the boy to identify himself with his model, and that this identification involves his reading himself consciously into the standpoint of his model, so that his own consciousness and that of his model, so far forth as that special series of activities is concerned, shall be the same. Now we have here, I think, an instructive example of the typical method by which the self comes into conscious relations with other selves and is able to form constructs of the selves which stand related to it as its social others. We are not dealing here with the practical motives that may 
enter into the situation and lead to actual association. Men as a matter of fact associate for all sorts of reasons. The question here is different. Assuming that men do and will associate for a variety of reasons, we ask: What is that fundamental quality of their nature which makes it possible for them to associate and without which association would be impossible? Mr. Spencer has given a general answer to this in the second volume of his 'Psychology,' in which he maintains that in order to sympathize with our fellows we must be able to represent to ourselves their consciousness and their actual mental condition. ${ }^{1}$ Now the whole theory of imitation may be regarded as a grounding of this general principle by showing how the representation of another's consciousness is achieved. And the analysis of the imitative situation has led us to expect that in it we have involved the most vital point of relationship between one individual consciousness and another. Let us endeavor then to make this clear. We have seen that a necessary condition of imitation is a model in the foreground of consciousness. The boy's model is his father planing and fitting floor boards. Only a part of this model is, however, an external representation. The most vital part for us is internal and consists in a construct which the boy has formed of the consciousness of his father. If now we scrutinize the situation with sufficient care we will find that the boy's construct of his father's consciousness which he has incorporated in his model is one that is defined just as far as his experience of his father enables him to define it, and beyond that it is undefined, or at least but vaguely guessed at. And the point of vital interest here is the fact that before the imitative activity begins just that part of the father's consciousness that is directly involved in the series of movements which the boy is trying to reproduce, will be an undefined region for the boy, and that the imitative movement wull have as its result its definition. Let us represent this part of the father's consciousness by $x$; it will be clear then that to the boy $x$ is an unknown quantity, and that the value of this quantity is to be determined by the experiment itself.

${ }^{1}$ Principles of Psycholorrs, Vol. II., Corollaries; I., Sociality and Sympathy. 
That $x$ shall be an unknown quantity is then an essential condition of the experiment. The boy is doubtless unaware of this, and he is least of all interested in a psychological experiment. All that he is conscious of is the fact that his model is interesting to him, and that there is a, to him, undefined impulse to attempt to realize it. Nevertheless he is taking part in a very profound experiment, and is putting both science and metaphysics under obligation. Let the problem here be to determine the value of $x$. Now the known terms are the present consciousness of the boy, which is undefined in its relation to $x$; the model which connects a series of movements with the father's consciousness, which to the boy is also undefined as respects $x^{x}$; and thirdly the impulse to imitation-that is, to a reproduction of the model. These are the known data. How then will the boy proceed to ascertain the value of $x$ ? The answer will be as follows. Obeying the impulse to imitate his model he will, no doubt in a very tentative way, proceed to perform the series of movements involved. He will provide himself with a carpenter's plane and with some pieces of flooring board, and will proceed to use the plane as he has seen it used, and finally to fit the pieces of board together so that the raised part of one will fit into the groove of the other, and he will no doubt prosecute the experiment until he has succeeded in obtaining a satisfactory result. This will represent the whole outward process, and will be all perhaps that the boy could give a very clear account of to his own consciousness. But in the meantime $x$ has not dropped out of the problem, and some very important steps have been taken in the determination of its value. For the boy has been learning how a carpenter feels in connection with his work, or this part of it, and in doing so has defined his own consciousness as respects the unknown term $x$. The value of $x$ expressed in terms of his own consciousness is the first-hand knowledge he has acquired of how the carpenterconsciousness operates in connection with this particular series of movements. We have then as the first step in the solution the determination of the value of $x$ for the boy's own consciousness. But it still remains to determine the value of $x$ for the father's consciousness. The peculiarity of the situation here is, of course, 
the fact that the father-consciousness is assumed already to know the value of $x$ for itself, and that the problem is altogether one for the consciousness of the boy. How shall the boy reach the construct of his father's consciousness so that he shall be able to sympathize with him in his work? It is clear that in order to discover the value of $x$ in the father's consciousness the boy must realize it in his own, and then using his own $x$-defined consciousness as a model he will by the use of the analogical reference construct a like defined consciousness for his father, and will assume that his father's conscious relation to his work will be the same as his own. And having thus determined the value of $x$ for his father's consciousness he will be able, taking the common value of $x$ as his basis, to enter sympathetically into his father's experience.

The above analysis of the situation has been followed out far enough to enable us to see clearly the modes by which one conscious self enters into and realizes the consciousness of another self. There is no magic involved, nor is the relation purely outward and extrinsic. But we find that, through the stimulus of the model in the foreground of consciousness, the boy, and his experience may here be generalized, enters upon a series of movements which enable him to effect a new definition in his own consciousness, and it is through this self-definition that he is able to form his construct of the consciousness of another. Now it is evident we may broaden out the situation beyond the limits of well-defined imitation, so as to include the direct as well as the indirect methods of interaction, and the principle will be the same. I mean by this that, whether we conceive the father as reacting directly upon the boy, or the boy as reacting directly upon his father, it will be true of these direct reactions, as it is of the indirect reactions in which imitation is overt, that each, in order to reach a construct of the consciousness of the other, must draw it up in terms of his own inner experience in similar relations. This brings the issue to a point where the last and most vital term in the theory of the social consciousness may be brought out and defined. We have seen that every step we take in construing the inner consciousness of another-that is, in conceiving the existence of 
another like ourselves-is preceded by the specific definition of our own self-consciousness in just the respect in which we proceed to define the other; and we have discovered this in connection with the fact that we were able to reach this definition, first of self and then of the other, through the medium of some common outer movement or series of movements, which we were able to relate to both self and the other as their common activities. Neglecting this latter feature for the present and taking into account only the inner relation between self-consciousness, and that of the other, it is clear that the condition of being innerly conscious of another self is the becoming ourselves conscious in the definite sense involved, and that it is from this definite self-consciousness that we form the construct or concept from which we read ourselves in to the consciousness of the other. The primacy of the self-consciousness is thus secured, and the consciousness of the other is, in this fundamental sense, its function. When, therefore, we ask either how the self comes to ascribe its analogies to another or how the other secures for itself a representation in the consciousness of the self and thus the power to influence it internally, the answer must be one in which this primacy is respected. For, whether we suppose that the consciousness in which the effect is to be produced has before it a definite model, as in explicit imitation, or simply certain outer signs which it interprets, we will find that the interpretation in either case will involve the bringing of the sign to the touchstone of some inner experience. Thus, when the child begins to cry on seeing her companion's finger bleed, the result is no immediate effect of the representation, but acquires its emotional power through some process that associates it with an inner experience of pain of the child's own, arising from an analogous cause. The touch that makes us kin is, therefore, an inner touch, while the objective and outer motive that leads to this touch is either an imitative movement or a representation that is rendered capable of a reference to the inner consciousness of another by means of its prior association with inner experiences of our own.

The conclusion of the whole matter may be stated in the following terms. We learn as the result of certain experiences 
to ascribe our inner consciousness, or its analogue, to others. The outward instruments of the development of this social consciousness are, broadly speaking, association and imitation. But when we pass from the consideration of the external instruments to that of the internal process we will find that we are able to enter into intelligible social relations with our other only because our nature is such that we are able to draw from the inner definitions of our own consciousness, brought about by certain objective agencies, a concept or construct of the consciousness of the other, which we conceive to be a true representation of his inner experience, and it is through this construct or representation that we are able to enter sympathetically into his life and treat him as a socius; a being like ourselves. On the other hand, if the question be how my other is able to come into social relations with me and to affect and modify me socially, the answer is very much the same. He can do so only by producing in me a definite representation of himself, and this is possible only through the outer mediation of association or imitation. The conditions are the same on both sides, and the truth is made clear that the only way in which social interaction is possible, or social effects producible, is through the power which each self-conscious individual has of internally representing the consciousness of his fellow; or, putting it from a different angle, the power which each self has of entering into the consciousness of its fellow and producing there an internal representation of itself. 